\title{
TWO-LEVEL ADDITIVE SCHWARZ PRECONDITIONERS FOR NONCONFORMING FINITE ELEMENT METHODS
}

\author{
SUSANNE C. BRENNER
}

\begin{abstract}
Two-level additive Schwarz preconditioners are developed for the nonconforming P1 finite element approximation of scalar second-order symmetric positive definite elliptic boundary value problems, the Morley finite element approximation of the biharmonic equation, and the divergence-free nonconforming P1 finite element approximation of the stationary Stokes equations. The condition numbers of the preconditioned systems are shown to be bounded independent of mesh sizes and the number of subdomains in the case of generous overlap.
\end{abstract}

\section{INTRODUCTION}

In this paper we develop two-level additive Schwarz preconditioners for the systems of linear equations resulting from nonconforming finite element approximations of elliptic boundary value problems. We obtain results with optimal convergence rate (i.e., the condition numbers of the preconditioned systems are uniformly bounded) when the overlap between subdomains is generous for the following three cases: (I) the P1 nonconforming finite element for the Laplace equation, (II) the Morley finite element for the biharmonic equation, and (III) the divergence-free P1 nonconforming finite element for the stationary Stokes equations. Our preconditioner is a variant of Dryja and Widlund's (cf. [10]) preconditioner for conforming finite element methods (cf. also [14]).

There is some recent work in this area for scalar second-order equations. Sarkis (cf. [15]) has developed a two-level additive Schwarz method using P1 nonconforming finite elements on both grids, which is insensitive to the jumps in coefficients but converges in a suboptimal rate. Cowsar (cf. [8]) has obtained the optimal convergence rate for a two-level additive Schwarz method using P1 nonconforming finite elements on the fine grid, but P1 conforming finite elements on the coarser grid.

In our approach, both the fine-grid and the coarse-grid spaces are nonconforming. The critical step is therefore the construction of intergrid transfer operators with certain properties. Our construction is based on the connection between the nonconforming finite element space and an appropriate conforming finite element

Received by the editor July 6, 1993 and, in revised form, November 18, 1993 and August 1, 1994.

1991 Mathematics Subject Classification. Primary 65F10, 65N30, 65N55.

Key words and phrases. Domain decomposition, additive Schwarz preconditioner, nonconforming finite elements, Laplace equation, biharmonic equation, stationary Stokes equations.

This work was supported in part by the National Science Foundation under Grant No. DMS92-09332.

(C)1996 American Mathematical Society 
space. For problems (I) and (II) we use the P2 conforming Lagrange finite element and the P5 Argyris finite element, respectively. Problem (III) is treated through the connection between the Morley finite element and the divergence-free P1 nonconforming finite element. The results in this paper were first announced in [3].

The rest of this paper is organized as follows. The abstract theory for scalar elliptic problems is developed in $\S 2$. We show in $\S \S 3$ and 4 that the abstract theory is applicable to problems (I) and (II) by constructing the intergrid transfer operators and verifying the assumptions of the abstract theory. In $\S 5$ the theory for scalar problems is modified and applied to the elliptic system of stationary Stokes equations.

Throughout the paper we use the following conventions for Sobolev norms and semi-norms of a function $v$ defined on an open set $G$ :

and

$$
\|v\|_{H^{m}(G)}:=\left(\int_{G} \sum_{|\alpha| \leq m}\left|\partial^{\alpha} v\right| d x\right)^{1 / 2}
$$

$$
|v|_{H^{m}(G)}:=\left(\int_{G} \sum_{|\alpha|=m}\left|\partial^{\alpha} v\right| d x\right)^{1 / 2} .
$$

We shall also denote the space of polynomials of degree less than or equal to $\ell$ on $G$ by $\mathcal{P}_{\ell}(G)$.

\section{Abstract theory}

Here we will develop a theory for scalar elliptic equations which satisfy homogeneous Dirichlet boundary conditions. Let $\Omega$ be a bounded polygonal domain in $\mathbb{R}^{2}$. We assume that $\Omega=\bigcup_{j=1}^{J} \Omega_{j}$, where $\Omega_{j}$ are open subdomains of $\Omega$. Let $\mathcal{T}_{H}$ be a quasi-uniform triangulation of $\Omega$ and $\mathcal{T}_{h}$ be a subdivision of $\mathcal{T}_{H}$ such that $\mathcal{T}_{h}$ is aligned with each $\partial \Omega_{j}$. The parameters $H$ and $h$ represent the mesh sizes. We assume that there exist nonnegative $C^{\infty}$ functions $\theta_{1}, \theta_{2}, \cdots, \theta_{J}$ in $\mathbb{R}^{2}$ such that

$$
\begin{aligned}
& \theta_{j}=0 \quad \text { on } \Omega \backslash \Omega_{j}, \\
& \sum_{j=1}^{J} \theta_{j}=1 \quad \text { on } \bar{\Omega}, \\
& \left\|\nabla \theta_{j}\right\|_{L^{\infty}} \leq \frac{C}{\delta}, \quad\left\|\nabla^{2} \theta_{j}\right\|_{L^{\infty}} \leq \frac{C}{\delta^{2}},
\end{aligned}
$$

where $\nabla^{2} \theta$ is the Hessian, $C$ is a universal constant and $\delta$ is a parameter, $0<h \leq$ $C_{1} \delta, 0<\delta \leq C_{2} H$. The constructions of $\Omega_{j}$ and $\theta_{j}$ are standard (cf. [10]). The parameter $\delta$ measures the amount of overlap among the subdomains $\Omega_{j}$. From now on, $C$ (with or without subscripts) will denote a generic positive constant independent of $h, H, \delta$, and $J$. We assume that there exists an integer $N_{c}$ independent of $h, H, \delta$, and $J$ such that any point in $\Omega$ can belong to at most $N_{c}$ subregions. We shall also assign the value 1 to the parameter $k$ for second-order problems, and the value 2 for fourth-order problems. 
Let $V_{h}$ be a finite element space associated with $\mathcal{T}_{h}$ whose members vanish at the boundary nodes, and $V_{j}$ be the subspace of $V_{h}$ whose members vanish at all nodes that are not interior to $\Omega_{j}$. The existence of the partition of unity $\theta_{1}, \theta_{2}, \cdots, \theta_{J}$ implies that

$$
V_{h}=\sum_{j=1}^{J} V_{j} .
$$

Also let $V_{H}$ be a finite element space associated with the triangulation $\mathcal{T}_{H}$ whose members vanish at the boundary nodes of $\Omega$. The members of $V_{h}$ and $V_{H}$ are piecewise polynomials of degree less than or equal to $k$.

The discretized problem is:

Find $u \in V_{h}$ such that

$$
a_{h}(u, v)=F(v) \quad \forall v \in V_{h},
$$

where $a_{h}(\cdot, \cdot)$ is a positive definite symmetric bilinear form on $V_{h}$ and $F \in V_{h}^{\prime}$. We assume that there is also a related positive definite bilinear form $a_{H}(\cdot, \cdot)$ defined on $V_{H}$.

For the description of the preconditioner we adopt the notation in [18]. Let $(\cdot, \cdot)_{h}$ and $(\cdot, \cdot)_{H}$ be two inner products on $V_{h}$ and $V_{H}$ respectively.

We define $A_{h}: V_{h} \longrightarrow V_{h}, A_{j}: V_{j} \longrightarrow V_{j}$ and $A_{H}: V_{H} \longrightarrow V_{H}$ by

$$
\begin{array}{cl}
\left(A_{h} v, w\right)_{h}=a_{h}(v, w) & \forall v, w \in V_{h}, \\
\left(A_{j} v, w\right)_{h}=a_{h}(v, w) & \forall v, w \in V_{j}, \\
\left(A_{H} v, w\right)_{H}=a_{H}(v, w) & \forall v, w \in V_{H} .
\end{array}
$$

The operators $Q_{j}: V_{h} \longrightarrow V_{j}, 1 \leq j \leq J$, are defined by

$$
\left(Q_{j} v, w\right)_{h}=(v, w)_{h} \quad \forall v \in V_{h}, w \in V_{j} .
$$

The operators $P_{j}: V_{h} \longrightarrow V_{j}, 1 \leq j \leq J$, are defined by

$$
a_{h}\left(P_{j} v, w\right)=a_{h}(v, w) \quad \forall v \in V_{h}, w \in V_{j} .
$$

It can be easily proved that

$$
A_{j} P_{j}=Q_{j} A_{h}, \quad 1 \leq j \leq J
$$

We assume that there is an intergrid transfer operator $I_{H}^{h}: V_{H} \longrightarrow V_{h}$ such that

$$
\begin{aligned}
\left|I_{H}^{h} v\right|_{H^{k}\left(\mathcal{T}_{h}\right)} & \leq C|v|_{H^{k}\left(\mathcal{T}_{H}\right)} \quad \forall v \in V_{H}, \\
\left|I_{H}^{h} v-v\right|_{H^{\ell}\left(\mathcal{T}_{h}\right)} & \leq C H^{k-\ell}|v|_{H^{k}\left(\mathcal{T}_{H}\right)} \quad \forall v \in V_{H}, 0 \leq \ell \leq k-1,
\end{aligned}
$$


where the (possibly) nonconforming norms $|\cdot|_{H^{m}\left(\mathcal{T}_{h}\right)}$ and $|\cdot|_{H^{m}\left(\mathcal{T}_{H}\right)}$ are defined by

$$
\begin{aligned}
|v|_{H^{m}\left(\mathcal{T}_{h}\right)} & :=\sqrt{\sum_{T \in \mathcal{T}_{h}}|v|_{H^{m}(T)}^{2}} \quad \forall v \in V_{h}, \\
|v|_{H^{m}\left(\mathcal{T}_{H}\right)} & :=\sqrt{\sum_{T \in \mathcal{T}_{H}}|v|_{H^{m}(T)}^{2}} \quad \forall v \in V_{H} .
\end{aligned}
$$

We also assume that

$$
\sqrt{a_{h}(v, v)}\left(\text { resp., } \sqrt{a_{H}(v, v)}\right) \text { is equivalent to }|v|_{H^{k}\left(\mathcal{T}_{h}\right)}\left(\text { resp., }|v|_{H^{k}\left(\mathcal{T}_{H}\right)}\right)
$$

for $v \in V_{h}$ (resp., $v \in V_{H}$ ), and

$$
a_{h}(v, w) \leq C|v|_{H^{k}\left(\mathcal{T}_{h, j}\right)}|w|_{H^{k}\left(\mathcal{T}_{h}\right)}
$$

for all $v \in V_{h}, w \in V_{j}$, where

$$
|v|_{H^{k}\left(\mathcal{T}_{h, j}\right)}=\left(\sum_{\substack{T \subset \Omega_{j} \\ T \in \mathcal{T}_{h}}}|v|_{H^{k}(T)}^{2}\right)^{1 / 2} .
$$

The operators $I_{h}^{H}, P_{h}^{H}: V_{h} \longrightarrow V_{H}$ are defined by

$$
\begin{aligned}
\left(I_{h}^{H} v, w\right)_{H} & =\left(v, I_{H}^{h} w\right)_{h} \quad \forall v \in V_{h}, w \in V_{H}, \\
a_{H}\left(P_{h}^{H} v, w\right) & =a_{h}\left(v, I_{H}^{h} w\right) \quad \forall v \in V_{h}, w \in V_{H} .
\end{aligned}
$$

In terms of operators, we can also express (2.13) as

$$
A_{H} P_{h}^{H}=I_{h}^{H} A_{h} .
$$

Also, (A.1a), (A.2a) and (2.13) imply that

$$
\left|P_{h}^{H} v\right|_{H^{k}\left(\mathcal{T}_{H}\right)} \leq C|v|_{H^{k}\left(\mathcal{T}_{h}\right)} \quad \forall v \in V_{h} .
$$

The two-level additive Schwarz preconditioner $B: V_{h} \longrightarrow V_{h}$ is defined by

$$
B:=I_{H}^{h} R_{H} I_{h}^{H}+\sum_{j=1}^{J} R_{j} Q_{j},
$$

where $R_{H}$ (resp., $R_{j}$ ) is an approximate solver of $A_{H}$ (resp., $A_{j}$ ) which is symmetric positive definite with respect to $(\cdot, \cdot)_{H}$ (resp., $\left.(\cdot, \cdot)_{h}\right)$.

The discretized problem (2.5) can be written as:

$$
A_{h} u=f
$$

where $F(v)=(f, v)_{h} \quad \forall v \in V_{h}$. 
The preconditioned system is:

$$
B A_{h} u=B f .
$$

The operator $B A_{h}$ is symmetric positive definite with respect to $a_{h}(\cdot, \cdot)$ because of the defining properties of the various operators and (2.4), and hence has positive eigenvalues $0<\lambda_{\min }\left(B A_{h}\right) \leq \cdots \leq \lambda_{\max }\left(B A_{h}\right)$. Our goal is to show that

$$
\frac{\lambda_{\max }\left(B A_{h}\right)}{\lambda_{\min }\left(B A_{h}\right)} \leq C
$$

By (2.11) and (2.14) we have

$$
\begin{aligned}
B A_{h} & =I_{H}^{h} R_{H} I_{h}^{H} A_{h}+\sum_{j=1}^{J} R_{j} Q_{j} A_{h} \\
& =I_{H}^{h} R_{H} A_{H} P_{h}^{H}+\sum_{j=1}^{J} R_{j} A_{j} P_{j} .
\end{aligned}
$$

Note that in the case where $R_{H}=A_{H}^{-1}$ and $R_{j}=A_{j}^{-1},(2.20)$ can be simplified to

$$
B A_{h}=I_{H}^{h} P_{h}^{H}+\sum_{j=1}^{J} P_{j}=\left(I_{H}^{h} A_{H}^{-1} I_{h}^{H}\right) A_{h}+\sum_{j=1}^{J} P_{j} .
$$

Comparing (2.21) with equation (13) in [15], we see that the two preconditioners are slightly different.

The techniques we use to bound the eigenvalues are based on the ideas of Dryja and Widlund in [10] and [11] (see also [18]). Their theory has also been extended by Zhang (cf. [19]) to fourth-order problems in the case of conforming finite elements. We begin with the upper bound for the eigenvalues of $B A_{h}$.

Lemma 2.1. The following inequality holds:

$$
\sum_{j=1}^{J} a_{h}\left(P_{j} v, P_{j} v\right) \leq C N_{c} a_{h}(v, v) \quad \forall v \in V_{h} .
$$

Proof. By (A.2) and (2.10) we have

$$
\begin{aligned}
a_{h}\left(P_{j} v, P_{j} v\right) & =a_{h}\left(v, P_{j} v\right) \\
& \leq C|v|_{H^{k}\left(\mathcal{T}_{h, j}\right)}\left|P_{j} v\right|_{H^{k}\left(\mathcal{T}_{h}\right)} \\
& \leq C|v|_{H^{k}\left(\mathcal{T}_{h, j}\right)} \sqrt{a_{h}\left(P_{j} v, P_{j} v\right)} .
\end{aligned}
$$

Therefore,

$$
a_{h}\left(P_{j} v, P_{j} v\right) \leq C|v|_{H^{k}\left(\mathcal{T}_{h, j}\right)}^{2}
$$


Summing over $1 \leq j \leq J$, we find by (A.2a) and the definition of $N_{c}$ that

$$
\sum_{j=1}^{J} a_{h}\left(P_{j} v, P_{j} v\right) \leq C N_{c}|v|_{H^{k}\left(\mathcal{T}_{h}\right)}^{2} \leq C N_{c} a_{h}(v, v) .
$$

Lemma 2.2. The following upper bound for the eigenvalues of $B A_{h}$ holds:

$$
\lambda_{\max }\left(B A_{h}\right) \leq C \omega_{1} N_{c},
$$

where $\omega_{1}:=\max \left(\rho\left(R_{H} A_{H}\right), \rho\left(R_{1} A_{1}\right), \cdots, \rho\left(R_{J} A_{J}\right)\right)$ and $\rho(\cdot)$ denotes the spectral radius.

Proof. By (2.20), (2.13), (2.15), (2.22) and (A.2a) we have

$$
\begin{aligned}
a_{h}\left(B A_{h} v, v\right) & =a_{h}\left(I_{H}^{h} R_{H} A_{H} P_{h}^{H} v, v\right)+\sum_{j=1}^{J} a_{h}\left(R_{j} A_{j} P_{j} v, v\right) \\
& =a_{H}\left(R_{H} A_{H} P_{h}^{H} v, P_{h}^{H} v\right)+\sum_{j=1}^{J} a_{h}\left(R_{j} A_{j} P_{j} v, P_{j} v\right) \\
& \leq \omega_{1}\left[a_{H}\left(P_{h}^{H} v, P_{h}^{H} v\right)+\sum_{j=1}^{J} a_{h}\left(P_{j} v, P_{j} v\right)\right] \\
& \leq \omega_{1}\left[C a_{h}(v, v)+C N_{c} a_{h}(v, v)\right] \\
& \leq C \omega_{1} N_{c} a_{h}(v, v) .
\end{aligned}
$$

In this derivation we have also used the fact that $R_{H} A_{H}$ (resp., $R_{j} A_{j}$ ) is symmetric positive definite with respect to $a_{H}(\cdot, \cdot)$ (resp., $\left.a_{h}(\cdot, \cdot)\right|_{V_{j}}$ ). The lemma follows immediately from (2.23).

We now turn our attention to the lower bound for the eigenvalues of $B A_{h}$. We assume that there exists an operator $J_{h}^{H}: V_{h} \longrightarrow V_{H}$ with the following properties:

$$
\begin{aligned}
\left|J_{h}^{H} v\right|_{H^{k}\left(\mathcal{T}_{H}\right)} & \leq C|v|_{H^{k}\left(\mathcal{T}_{h}\right)} \quad \forall v \in V_{h}, \\
\left|J_{h}^{H} v-v\right|_{H^{\ell}\left(\mathcal{T}_{h}\right)} & \leq C H^{k-\ell}|v|_{H^{k}\left(\mathcal{T}_{h}\right)} \quad \forall v \in V_{h}, 0 \leq \ell \leq k-1 .
\end{aligned}
$$

Note that in our theory, the finite element spaces $V_{h}$ and $V_{H}$ are connected by the operators $I_{H}^{h}, J_{h}^{H},(\mathrm{~A} .1)$ and (A.3), but only $I_{H}^{h}$ appears in the preconditioner.

Let $\Pi_{h}$ be the nodal interpolation operator associated with $\mathcal{T}_{h}$. We assume that

$$
\left|\Pi_{h}(\lambda v)\right|_{H^{k}(T)} \leq C|\lambda v|_{H^{k}(T)} \quad \forall T \in \mathcal{T}_{h}, v \in \mathcal{P}_{k}(T), \lambda \in \mathcal{P}_{k-1}(T)
$$

and

$$
\left\|\Pi_{h}(g v)\right\|_{L^{2}(T)} \leq C\left(\|g\|_{L^{\infty}(T)}+(k-1) h\|\nabla g\|_{L^{\infty}(T)}\right)\|v\|_{L^{2}(T)}
$$

$\forall T \in \mathcal{T}_{h}, v \in \mathcal{P}_{k}(T), g \in C^{\infty}(\bar{T})$, where $C$ only depends on the minimum angle in $\mathcal{T}_{h}$. 
Lemma 2.3. Given any $v \in V_{h}$, there exists $v_{0} \in V_{H}, v_{j} \in V_{j}(1 \leq j \leq J)$ such that

$$
v=I_{H}^{h} v_{0}+\sum_{j=1}^{J} v_{j}
$$

and

$$
a_{H}\left(v_{0}, v_{0}\right)+\sum_{j=1}^{J} a_{h}\left(v_{j}, v_{j}\right) \leq C N_{c}\left(1+\left(\frac{H}{\delta}\right)^{2 k}\right) a_{h}(v, v) .
$$

Proof. Let $v_{0}=J_{h}^{H} v$ and $v_{j}=\Pi_{h}\left(\theta_{j}\left(v-I_{H}^{h} v_{0}\right)\right)$, where $\Pi_{h}$ is the nodal variable interpolation operator associated with $V_{h}$. Clearly, (2.24) holds.

We treat the cases $k=1$ and $k=2$ separately. For $k=1$, let $\bar{\theta}_{j, T}=\frac{1}{|T|} \int_{T} \theta_{j} d x$ for all $T \in \mathcal{T}_{h}$. Then we have by a straightforward computation that

$$
\left\|\theta_{j}-\bar{\theta}_{j, T}\right\|_{L^{\infty}(T)} \leq h\left\|\nabla \theta_{j}\right\|_{L^{\infty}(T)} .
$$

Let $w=v-I_{H}^{h} v_{0}$. Then by the triangle inequality, a standard inverse estimate (cf. $[7,6]),($ A.4), (2.26), (2.2) and (2.3) we have

$$
\begin{aligned}
\left|v_{j}\right|_{H^{1}(T)} & =\left|\Pi_{h}\left(\theta_{j} w\right)\right|_{H^{1}(T)} \\
& \leq\left|\bar{\theta}_{j, T} w\right|_{H^{1}(T)}+\left|\Pi_{h}\left[\left(\theta_{j}-\bar{\theta}_{j, T}\right) w\right]\right|_{H^{1}(T)} \\
& \leq|w|_{H^{1}(T)}+C h^{-1}\left\|\Pi_{h}\left[\left(\theta_{j}-\bar{\theta}_{j, T}\right) w\right]\right\|_{L^{2}(T)} \\
& \leq|w|_{H^{1}(T)}+C h^{-1}\left\|\theta_{j}-\bar{\theta}_{j, T}\right\|_{L^{\infty}(T)}\|w\|_{L^{2}(T)} \\
& \leq|w|_{H^{1}(T)}+\frac{C}{\delta}\|w\|_{L^{2}(T)} .
\end{aligned}
$$

Summing the square of (2.27) over $T$ in $\Omega_{j}$, we find by (A.2b) that

$$
a_{h}\left(v_{j}, v_{j}\right) \leq C\left(|w|_{H^{1}\left(\mathcal{T}_{h, j}\right)}^{2}+\frac{1}{\delta^{2}}\|w\|_{L^{2}\left(\Omega_{j}\right)}^{2}\right) .
$$

Summing (2.28) for $1 \leq j \leq J$, we obtain

$$
\sum_{j=1}^{J} a_{h}\left(v_{j}, v_{j}\right) \leq C N_{c}\left(a_{h}(w, w)+\frac{1}{\delta^{2}}\|w\|_{L^{2}(\Omega)}^{2}\right) .
$$

By (A.1a) and (A.3a) we have

$$
\begin{aligned}
|w|_{H^{1}\left(\mathcal{T}_{h}\right)} & =\left|v-I_{H}^{h} v_{0}\right|_{H^{1}\left(\mathcal{T}_{h}\right)} \\
& \leq|v|_{H^{1}\left(\mathcal{T}_{h}\right)}+\left|I_{H}^{h} v_{0}\right|_{H^{1}\left(\mathcal{T}_{h}\right)} \\
& \leq|v|_{H^{1}\left(\mathcal{T}_{h}\right)}+C\left|v_{0}\right|_{H^{1}\left(\mathcal{T}_{H}\right)} \\
& =|v|_{H^{1}\left(\mathcal{T}_{h}\right)}+C\left|J_{h}^{H} v\right|_{H^{1}\left(\mathcal{T}_{H}\right)} \\
& \leq C|v|_{H^{1}\left(\mathcal{T}_{h}\right)}
\end{aligned}
$$


which together with (A.2a) imply that

$$
a_{h}(w, w) \leq C a_{h}(v, v) .
$$

On the other hand, by (A.3b), (A.1b), (A.3a) and (A.2a),

$$
\begin{aligned}
\|w\|_{L^{2}(\Omega)} & =\left\|v-I_{H}^{h} J_{h}^{H} v\right\|_{L^{2}(\Omega)} \\
& \leq\left\|v-J_{h}^{H} v\right\|_{L^{2}(\Omega)}+\left\|J_{h}^{H} v-I_{H}^{h} J_{h}^{H} v\right\|_{L^{2}(\Omega)} \\
& \leq C H|v|_{H^{1}\left(\mathcal{T}_{h}\right)}+C H\left|J_{h}^{H} v\right|_{H^{1}\left(\mathcal{T}_{H}\right)} \\
& \leq C H|v|_{H^{1}\left(\mathcal{T}_{h}\right)} \\
& \leq C H \sqrt{a_{h}(v, v)} .
\end{aligned}
$$

Similarly, by (A.2a) and (A.3a),

$$
a_{H}\left(v_{0}, v_{0}\right) \leq C a_{h}(v, v) .
$$

Inequality (2.25) now follows from (2.29)-(2.32).

For $k=2$, let $\tilde{\theta}_{j, T}$ be the linear interpolant of $\theta_{j}$ on $T$, i.e., $\tilde{\theta}_{j, T} \in \mathcal{P}_{1}(T)$ and $\tilde{\theta}_{j, T}=\theta_{j}$ at the vertices of $T$. It is clear that

$$
\left\|\tilde{\theta}_{j, T}\right\|_{L^{\infty}(T)} \leq\left\|\theta_{j}\right\|_{L^{\infty}(T)} \quad \text { and } \quad\left\|\nabla \tilde{\theta}_{j, T}\right\|_{L^{\infty}(T)} \leq C\left\|\nabla \theta_{j}\right\|_{L^{\infty}(T)},
$$

where $C$ depends only on the minimum angle of the triangulation $\mathcal{T}_{h}$.

By a simple homogeneity (scaling) argument we also have

$$
\begin{aligned}
& \left\|\theta_{j}-\tilde{\theta}_{j, T}\right\|_{L^{\infty}(T)}+h\left\|\nabla\left(\theta_{j}-\tilde{\theta}_{j, T}\right)\right\|_{L^{\infty}(T)} \\
& \leq C h^{2}\left\|\nabla^{2} \theta_{j}\right\|_{L^{\infty}(T)},
\end{aligned}
$$

where $C$ again depends only on the minimum angle of the triangulation $\mathcal{T}_{h}$.

Let $w=v-I_{H}^{h} v_{0}$. Then by the triangle inequality, a standard inverse estimate, (A.4), (2.33), (2.34) and (2.3) we have

$$
\begin{aligned}
\left|v_{j}\right|_{H^{2}(T)}= & \left|\Pi_{h}\left(\theta_{j} w\right)\right|_{H^{2}(T)} \\
\leq & \left|\Pi_{h}\left(\tilde{\theta}_{j, T} w\right)\right|_{H^{2}(T)}+\left|\Pi_{h}\left[\left(\theta_{j}-\tilde{\theta}_{j, T}\right) w\right]\right|_{H^{2}(T)} \\
\leq & C\left|\tilde{\theta}_{j, T} w\right|_{H^{2}(T)}+C h^{-2}\left\|\Pi_{h}\left[\left(\theta_{j}-\tilde{\theta}_{j, T}\right) w\right]\right\|_{L^{2}(T)} \\
\leq & C\left(\left\|\tilde{\theta}_{j, T}\right\|_{L^{\infty}(T)}|w|_{H^{2}(T)}+\left\|\nabla \tilde{\theta}_{j, T}\right\|_{L^{\infty}(T)}|w|_{H^{1}(T)}\right) \\
& +C h^{-2}\left(\left\|\theta_{j}-\tilde{\theta}_{j, T}\right\|_{L^{\infty}(T)}+h\left\|\nabla\left(\theta_{j}-\tilde{\theta}_{j, T}\right)\right\|_{L^{\infty}(T)}\right)\|w\|_{L^{2}(T)} \\
\leq & C\left(|w|_{H^{2}(T)}+\frac{1}{\delta}|w|_{H^{1}(T)}+\frac{1}{\delta^{2}}\|w\|_{L^{2}(T)}\right) .
\end{aligned}
$$

Summing up the square of (2.35) over $T$ in $\Omega_{j}$, we find by (A.2b) that

$$
a_{h}\left(v_{j}, v_{j}\right) \leq C\left(|w|_{H^{2}\left(\mathcal{T}_{h, j}\right)}^{2}+\frac{1}{\delta^{2}}|w|_{H^{1}\left(\mathcal{T}_{h, j}\right)}^{2}+\frac{1}{\delta^{4}}\|w\|_{L^{2}\left(\Omega_{j}\right)}^{2}\right) .
$$


Summing up (2.36) for $1 \leq j \leq J$, we obtain

$$
\sum_{j=1}^{J} a_{h}\left(v_{j}, v_{j}\right) \leq C N_{c}\left(a_{h}(w, w)+\frac{1}{\delta^{2}}|w|_{H^{1}\left(\mathcal{T}_{h}\right)}^{2}+\frac{1}{\delta^{4}}\|w\|_{L^{2}(\Omega)}^{2}\right) .
$$

As in the previous case we deduce from (A.1a), (A.2), and (A.3a) that

$$
a_{h}(w, w) \leq C a_{h}(v, v)
$$

and

$$
a_{H}\left(v_{0}, v_{0}\right) \leq C a_{h}(v, v) .
$$

Also, analogous to (2.31), we have by (A.1), (A.2) and (A.3) that

$$
\|w\|_{L^{2}(\Omega)}+H|w|_{H^{1}\left(\mathcal{T}_{h}\right)} \leq C H^{2} \sqrt{a_{h}(v, v)} .
$$

Inequality (2.25) now follows by combining (2.37)-(2.40).

Lemma 2.4. The following lower bound for the eigenvalues of $B A_{h}$ holds:

$$
\lambda_{\min }\left(B A_{h}\right) \geq C \frac{\omega_{0}}{N_{c}\left(1+\left(\frac{H}{\delta}\right)^{2 k}\right)},
$$

where $\omega_{0}:=\min \left(\lambda_{\min }\left(R_{H} A_{H}\right), \lambda_{\min }\left(R_{1} A_{1}\right), \cdots, \lambda_{\min }\left(R_{J} A_{J}\right)\right)$.

Proof. Let $\beta:=N_{c}\left(1+\left(\frac{H}{\delta}\right)^{2 k}\right)$. Given any $v \in V_{h}$, by Lemma 2.3 there exists $v_{0} \in V_{H}, v_{j} \in V_{j}(1 \leq j \leq J)$ such that (2.24) and (2.25) hold. It follows from (2.25) that

$$
\begin{aligned}
C \beta a_{h}(v, v) & \geq a_{H}\left(v_{0}, v_{0}\right)+\sum_{j=1}^{J} a_{h}\left(v_{j}, v_{j}\right) \\
& =\left(R_{H}^{-1} R_{H} A_{H} v_{0}, v_{0}\right)_{H}+\sum_{j=1}^{J}\left(R_{j}^{-1} R_{j} A_{j} v_{j}, v_{j}\right)_{h} \\
& \geq \omega_{0}\left[\left(R_{H}^{-1} v_{0}, v_{0}\right)_{H}+\sum_{j=1}^{J}\left(R_{j}^{-1} v_{j}, v_{j}\right)_{h}\right]
\end{aligned}
$$

where we have used the fact that $R_{H} A_{H}: V_{H} \longrightarrow V_{H}$ (resp., $R_{j} A_{j}: V_{j} \longrightarrow V_{j}$ ) is positive definite with respect to $\left(R_{H}^{-1} \cdot, \cdot\right)_{H}$ (resp., $\left.\left(R_{j}^{-1} \cdot, \cdot\right)_{h}\right)$.

Let $T_{H}:=I_{H}^{h} R_{H} A_{H} P_{h}^{H}$ and $T_{j}:=R_{j} A_{j} P_{j}$. In other words, we can rewrite $(2.20)$ as

$$
B A_{h}=T_{H}+\sum_{j=1}^{J} T_{j}
$$

Using (2.24), (2.13), (2.10), the Cauchy-Schwarz inequality, (2.42) and (2.43), we 
have

$$
\begin{aligned}
a_{h}(v, v)= & a_{h}\left(I_{H}^{h} v_{0}, v\right)+\sum_{j=1}^{J} a_{h}\left(v_{j}, v\right) \\
= & a_{H}\left(v_{0}, P_{h}^{H} v\right)+\sum_{j=1}^{J} a_{h}\left(v_{j}, P_{j} v\right) \\
= & \left(R_{H}^{-1 / 2} v_{0}, R_{H}^{1 / 2} A_{H} P_{h}^{H} v\right)_{H}+\sum_{j=1}^{J}\left(R_{j}^{-1 / 2} v_{j}, R_{j}^{1 / 2} A_{j} P_{j} v\right)_{h} \\
\leq & \left(R_{H}^{-1} v_{0}, v_{0}\right)_{H}^{1 / 2}\left(A_{H} P_{h}^{H} v, R_{H} A_{H} P_{h}^{H} v\right)_{H}^{1 / 2} \\
& +\sum_{j=1}^{J}\left(R_{j}^{-1} v_{j}, v_{j}\right)_{h}^{1 / 2}\left(A_{j} P_{j} v, R_{j} A_{j} P_{j} v\right)_{h}^{1 / 2} \\
= & \left(R_{H}^{-1} v_{0}, v_{0}\right)_{H}^{1 / 2} a_{H}\left(P_{h}^{H} v, R_{H} A_{H} P_{h}^{H} v\right)^{1 / 2} \\
& +\sum_{j=1}^{J}\left(R_{j}^{-1} v_{j}, v_{j}\right)_{h}^{1 / 2} a_{h}\left(P_{j} v, R_{j} A_{j} P_{j} v\right)^{1 / 2} \\
= & \left(R_{H}^{-1} v_{0}, v_{0}\right)_{H}^{1 / 2} a_{h}\left(v, T_{H} v\right)^{1 / 2}+\sum_{j=1}^{J}\left(R_{j}^{-1} v_{j}, v_{j}\right)_{h}^{1 / 2} a_{h}\left(v, T_{j} v\right)^{1 / 2} \\
\leq & \left(\left(R_{H}^{-1} v_{0}, v_{0}\right)_{H}+\sum_{j=1}^{J}\left(R_{j}^{-1} v_{j}, v_{j}\right)_{h}\right)^{1 / 2}\left(a_{h}\left(v, T_{H} v\right)+\sum_{j=1}^{J} a_{h}\left(v, T_{j} v\right)\right)^{1 / 2} \\
\leq & \frac{C \beta^{1 / 2}}{\omega_{0}^{1 / 2} a_{h}(v, v)^{1 / 2} a_{h}\left(v, B A_{h} v\right)^{1 / 2},}
\end{aligned}
$$

which implies that

$$
a_{h}(v, v) \leq \frac{C \beta}{\omega_{0}} a_{h}\left(v, B A_{h} v\right) .
$$

Inequality (2.41) now follows immediately from (2.44).

In summary, we have the following theorem.

Theorem 2.1. Under the geometric assumptions (2.1)-(2.3) and the assumptions (A.1)-(A.4) on the finite element spaces we have

$$
\frac{\lambda_{\max }\left(B A_{h}\right)}{\lambda_{\min }\left(B A_{h}\right)} \leq C \frac{\omega_{1}}{\omega_{0}} N_{c}^{2}\left(1+\left(\frac{H}{\delta}\right)^{2 k}\right) .
$$

Therefore, if the approximate solvers $R_{H}$ and $R_{j}$ are accurate enough so that $\omega_{1}$ is bounded and $\omega_{0}$ is bounded away from zero, and if the overlap between subregions is generous enough so that $\frac{H}{\delta}$ is bounded, then the condition number of the preconditioned system is bounded independent of $h, \delta, H$ and $J$.

Remark. If we are more careful about the definition of $\Omega_{j}$ and $\theta_{j}$, then the factor $1+\left(\frac{H}{\delta}\right)^{2 k}$ of Theorem 2.1 can be reduced to $1+\left(\frac{H}{\delta}\right)^{2 k-1}$. This is done by using a trace theorem type argument in [17], which can be applied to nonconforming finite elements after a slight modification (cf. [4]). 


\section{SCALAR P1 nONCONFORMing Finite ELEMENT}

In this section we apply the abstract theory to the P1 nonconforming finite element (cf. [9]) approximation of the Laplace equation. The finite element space $V_{h}$ is defined by

$$
\begin{aligned}
V_{h}:= & \left\{v \in L^{2}(\Omega): v \in \mathcal{P}_{1}(T) \forall T \in \mathcal{T}_{h}, v\right. \text { is continuous at the } \\
& \text { midpoints of interelement boundaries, and } v \text { vanishes } \\
& \text { at the midpoints along } \partial \Omega\} .
\end{aligned}
$$

$V_{H}$ is defined the same way with respect to $\mathcal{T}_{H}$. Members of $V_{h}$ (resp., $V_{H}$ ) are completely determined by their values at the midpoints of $\mathcal{T}_{h}$ (resp., $\mathcal{T}_{H}$ ).

The symmetric positive definite bilinear forms $a_{h}(\cdot, \cdot)$ and $a_{H}(\cdot, \cdot)$ are given by

$$
a_{h}\left(v_{1}, v_{2}\right):=\sum_{T \in \mathcal{T}_{h}} \int_{T} \nabla v_{1} \cdot \nabla v_{2} d x \quad \forall v_{1}, v_{2} \in V_{h}
$$

and

$$
a_{H}\left(v_{1}, v_{2}\right):=\sum_{T \in \mathcal{T}_{H}} \int_{T} \nabla v_{1} \cdot \nabla v_{2} d x \quad \forall v_{1}, v_{2} \in V_{H} .
$$

The inner products $(\cdot, \cdot)_{h}$ and $(\cdot, \cdot)_{H}$ are just the $L^{2}$-inner products restricted to $V_{h}$ and $V_{H}$, respectively. Note that the natural nodal basis functions in $V_{h}$ are $L^{2}$-orthogonal, so that the constructions of the $Q_{j}$ are trivial.

Assumptions (A.2) and (A.4a) are trivially satisfied, while (A.4b) follows from the following quadrature formula:

$$
\int_{T} v^{2} d x=\frac{|T|}{3}\left[\left(v\left(m_{1}\right)\right)^{2}+\left(v\left(m_{2}\right)\right)^{2}+\left(v\left(m_{3}\right)\right)^{2}\right] \quad \forall v \in \mathcal{P}_{1}(T),
$$

where $m_{1}, m_{2}$, and $m_{3}$ are the midpoints of the three sides of the triangle $T$.

It only remains to define the operators $I_{H}^{h}$ and $J_{h}^{H}$, and to verify assumptions (A.1a), (A.1b), (A.3a), and (A.3b). We introduce two other finite element spaces $W_{h}$ and $W_{H}$, where

$$
W_{h}:=\left\{w \in C(\bar{\Omega}):\left.w\right|_{T} \in \mathcal{P}_{2}(T) \quad \forall T \in \mathcal{T}_{h} \text { and } w=0 \text { on } \partial \Omega\right\},
$$

and $W_{H}$ is defined similarly with respect to $\mathcal{T}_{H}$. The members of $W_{h}$ (resp., $W_{H}$ ) are completely determined by their values at the vertices and midpoints of $\mathcal{T}_{h}$ (resp., $\left.\mathcal{T}_{H}\right)$. Note that $W_{H} \subset W_{h}$ since $\mathcal{T}_{h}$ is a subdivision of $\mathcal{T}_{H}$.

We define $E_{h}: V_{h} \longrightarrow W_{h}$ and $F_{h}: W_{h} \longrightarrow V_{h}$ by

$$
\begin{cases}\left(E_{h} v\right)(m)=v(m) & \text { for all internal midpoints } m \in \mathcal{T}_{h} \\ \left(E_{h} v\right)(p)=\text { average of } v_{i}(p) & \text { for all internal vertices } p \in \mathcal{T}_{h}\end{cases}
$$

where $v_{i}=\left.v\right|_{T_{i}}$ and $T_{i} \in \mathcal{T}_{h}$ contains $p$ as a vertex, and

$$
\left(F_{h} w\right)(m)=w(m) \quad \text { for all midpoints } m \in \mathcal{T}_{h} .
$$


The operators $E_{H}: V_{H} \longrightarrow W_{H}$ and $F_{H}: W_{H} \longrightarrow V_{H}$ are defined similarly with respect to $\mathcal{T}_{H}$.

The intergrid transfer operator $I_{H}^{h}: V_{H} \longrightarrow V_{h}$ is given by

$$
I_{H}^{h}:=F_{h} \circ E_{H} .
$$

The operator $J_{h}^{H}: V_{h} \longrightarrow V_{H}$ is given by

$$
J_{h}^{H}:=F_{H} \circ Q_{h}^{H} \circ E_{h},
$$

where $Q_{h}^{H}: W_{h} \longrightarrow W_{H}$ is the $L^{2}$-orthogonal projection operator. The relations of these operators are illustrated by the commutative diagrams in Fig. 1 (where $i$ stands for natural injection).
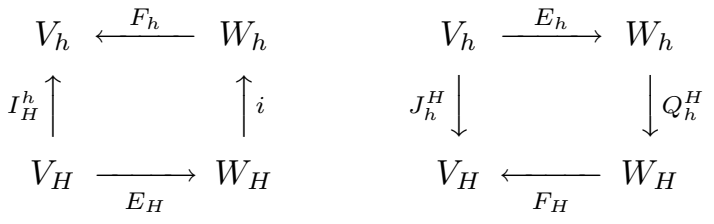

FiguRE 1

Note that $I_{H}^{h}$ is represented by a sparse, banded matrix with respect to the natural nodal bases of $V_{h}$ and $V_{H}$.

The estimates (A.1) and (A.3) are established through a sequence of lemmas. The next lemma follows from the result of Bramble and $\mathrm{Xu}$ in [2].

Lemma 3.1. The following estimates on $Q_{h}^{H}$ hold:

$$
\begin{aligned}
\left|Q_{h}^{H} w\right|_{H^{1}(\Omega)} & \leq C|w|_{H^{1}(\Omega)} \quad \forall w \in W_{h}, \\
\left\|w-Q_{h}^{H} w\right\|_{L^{2}(\Omega)} & \leq C H|w|_{H^{1}(\Omega)} \quad \forall w \in W_{h} .
\end{aligned}
$$

Lemma 3.2. The following estimates on $F_{h}$ and $F_{H}$ hold:

$$
\begin{aligned}
\left|F_{h} w\right|_{H^{1}\left(\mathcal{T}_{h}\right)} \leq C|w|_{H^{1}(\Omega)} \quad \forall w \in W_{h}, \\
\left|F_{H} w\right|_{H^{1}\left(\mathcal{T}_{H}\right)} \leq C|w|_{H^{1}(\Omega)} \quad \forall w \in W_{H}, \\
\left\|w-F_{h} w\right\|_{L^{2}(\Omega)} \leq C h|w|_{H^{1}(\Omega)} \quad \forall w \in W_{h}, \\
\left\|w-F_{H} w\right\|_{L^{2}(\Omega)} \leq C H|w|_{H^{1}(\Omega)} \quad \forall w \in W_{H} .
\end{aligned}
$$

Proof. It suffices to establish (3.9a) and (3.10a). On a reference triangle $\hat{T},|\cdot|_{H^{1}(\hat{T})}$ defines a norm on the quotient space $\mathcal{P}_{2}(\hat{T}) / \mathcal{P}_{0}(\hat{T})$. Given any $w \in \mathcal{P}_{2}(\hat{T})$, let $w^{\prime} \in \mathcal{P}_{1}(\hat{T})$ be defined by $w^{\prime}\left(m_{i}\right)=w\left(m_{i}\right)$ at the midpoints $m_{i}(i=1,2,3)$ of $\hat{T}$. Since $w^{\prime}=w$ if $w \in \mathcal{P}_{0}(\hat{T}), w \longrightarrow w-w^{\prime}$ is a well-defined linear map from $\mathcal{P}_{2}(\hat{T}) / \mathcal{P}_{0}(\hat{T})$ into $\mathcal{P}_{2}(\hat{T})$. Therefore we have

$$
\left\|w-w^{\prime}\right\|_{L^{2}(\hat{T})} \leq C|w|_{H^{1}(\hat{T})} \quad \forall w \in \mathcal{P}_{2}(\hat{T}) .
$$




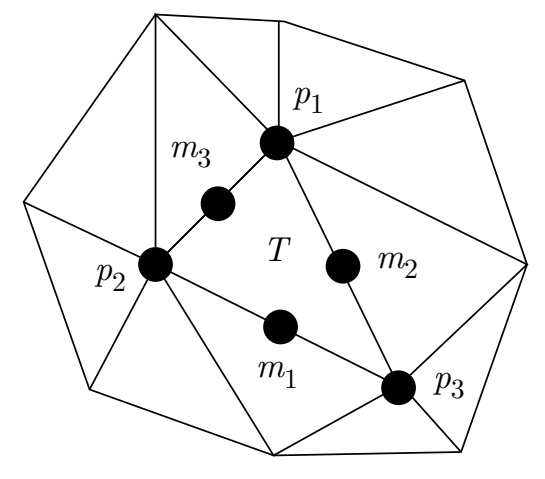

FIGURE 2

The estimate (3.11) together with a homogeneity argument yields

$$
\left\|w-F_{h} w\right\|_{L^{2}(\Omega)} \leq C h|w|_{H^{1}(\Omega)} \quad \forall w \in W_{h},
$$

where $C$ depends only on the minimum angle of $\mathcal{T}_{h}$.

By a standard inverse estimate and (3.12), we obtain

$$
\begin{aligned}
\left|F_{h} w\right|_{H^{1}\left(\mathcal{T}_{h}\right)} & \leq\left|w-F_{h} w\right|_{H^{1}\left(\mathcal{T}_{h}\right)}+|w|_{H^{1}\left(\mathcal{T}_{h}\right)} \\
& \leq C h^{-1}\left\|w-F_{h} w\right\|_{L^{2}\left(\mathcal{T}_{h}\right)}+|w|_{H^{1}\left(\mathcal{T}_{h}\right)} \\
& \leq C|w|_{H^{1}(\Omega)} . \quad \square
\end{aligned}
$$

Lemma 3.3. The following estimates on $E_{h}$ and $E_{H}$ hold:

$$
\begin{aligned}
\left|E_{h} v\right|_{H^{1}(\Omega)} & \leq C|v|_{H^{1}\left(\mathcal{T}_{h}\right)} \quad \forall v \in V_{h}, \\
\left|E_{H} v\right|_{H^{1}(\Omega)} & \leq C|v|_{H^{1}\left(\mathcal{T}_{H}\right)} \quad \forall v \in V_{H}, \\
\left\|v-E_{h} v\right\|_{L^{2}(\Omega)} & \leq C h|v|_{H^{1}\left(\mathcal{T}_{h}\right)} \quad \forall v \in V_{h}, \\
\left\|v-E_{H} v\right\|_{L^{2}(\Omega)} & \leq C H|v|_{H^{1}\left(\mathcal{T}_{H}\right)} \quad \forall v \in V_{H} .
\end{aligned}
$$

Proof. It suffices to establish (3.13a) and (3.14a). Observe that $F_{h} \circ E_{h}=$ Id. Hence by (3.9a) we have

$$
\begin{aligned}
\left\|v-E_{h} v\right\|_{L^{2}(\Omega)} & =\left\|F_{h}\left(E_{h} v\right)-E_{h} v\right\|_{L^{2}(\Omega)} \\
& \leq C h\left|E_{h} v\right|_{H^{1}(\Omega)} .
\end{aligned}
$$

In view of (3.15), the whole problem is reduced to proving (3.13a).

Let $T \in \mathcal{T}_{h}$ be a triangle away from $\partial \Omega$, and $G$ be the union of all triangles in $\mathcal{T}_{h}$ sharing a vertex with $T$ (cf. Fig. 2). (The triangle $T$ is itself in $G$.) Let $V_{G}=\left\{v \in L^{2}(G):\left.v\right|_{T} \in \mathcal{P}_{1}(T) \quad \forall T \subset G, v\right.$ is continuous at the midpoints of the interelement boundaries $\}$.

Given $v \in V_{G}$, let $v^{\prime} \in \mathcal{P}_{2}(T)$ be defined by

$$
\begin{cases}v^{\prime}\left(m_{i}\right)=v\left(m_{i}\right) & \text { for } i=1,2,3 \\ v^{\prime}\left(p_{i}\right)=\text { average of } v_{i j} \text { at } p_{i} & \text { for } i=1,2,3\end{cases}
$$

where $v_{i j}=\left.v\right|_{T_{j}}$ and $T_{j} \subset G$ contains $p_{i}$ as a vertex. 

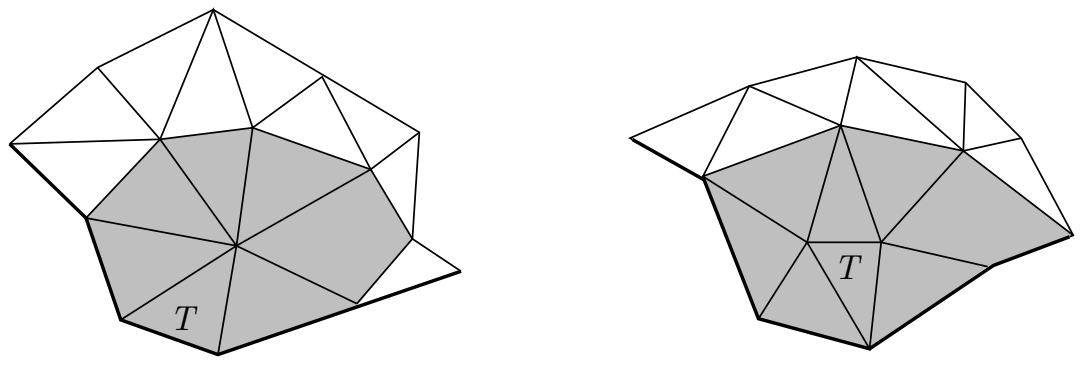

Figure 3

Let $|\cdot|_{H_{*}^{1}(G)}$ be defined by

$$
|v|_{H_{*}^{1}(G)}=\left(\sum_{T \subset G}|v|_{H^{1}(T)}^{2}\right)^{1 / 2} \quad \forall v \in V_{G}
$$

Observe that

$$
\begin{aligned}
|v|_{H_{*}^{1}(G)}=0 & \Longrightarrow v \in \mathcal{P}_{0}(G) \\
& \Longrightarrow v^{\prime}=v \quad \text { on } T \\
& \Longrightarrow\left|v^{\prime}\right|_{H^{1}(T)}=0 .
\end{aligned}
$$

It follows from (3.16) and a homogeneity argument using reference triangles that

$$
\left|v^{\prime}\right|_{H^{1}(T)} \leq C_{1}|v|_{H_{*}^{1}(G)} \quad \forall v \in V_{G},
$$

where $C$ depends on the number of triangles in $G$ and the shape of the triangles in $G$. Since $\mathcal{T}_{h}$ is quasi-uniform, $C_{1}$ ultimately depends on the minimum angle in $\mathcal{T}_{h}$.

The same estimate holds if the triangle $T$ is close to $\partial \Omega$, in which case the members of $V_{G}$ will vanish at certain midpoints (cf. Fig. 3).

Summing up the square of (3.17) over all triangles $T \in \mathcal{T}_{h}$, we obtain (3.13a).

Proposition 3.1. Assumptions (A.1) and (A.3) hold for $I_{H}^{h}$ and $J_{h}^{H}$ defined by (3.5) and (3.6), respectively.

Proof. The estimates (A.1a) and (A.3a) follow immediately from the estimates (3.7), (3.9) and (3.13).

Using (3.10a), (3.14a), and (3.13b), we have

$$
\begin{aligned}
\left\|I_{H}^{h} v-v\right\|_{L^{2}(\Omega)} & =\left\|F_{h}\left(E_{H} v\right)-v\right\|_{L^{2}(\Omega)} \\
& \leq\left\|F_{h}\left(E_{H} v\right)-E_{H} v\right\|_{L^{2}(\Omega)}+\left\|E_{H} v-v\right\|_{L^{2}(\Omega)} \\
& \leq C h\left|E_{H} v\right|_{H^{1}(\Omega)}+C H|v|_{H^{1}\left(\mathcal{T}_{H}\right)} \\
& \leq C H|v|_{H^{1}\left(\mathcal{T}_{H}\right)} .
\end{aligned}
$$


Similarly, using (3.10b), (3.8), (3.14a), (3.7), and (3.13a), we obtain

$$
\begin{aligned}
\left\|J_{h}^{H} v-v\right\|_{L^{2}(\Omega)}= & \left\|F_{H}\left(Q_{h}^{H}\left(E_{h} v\right)\right)-v\right\|_{L^{2}(\Omega)} \\
\leq & \left\|F_{H}\left(Q_{h}^{H}\left(E_{h} v\right)\right)-Q_{h}^{H}\left(E_{h} v\right)\right\|_{L^{2}(\Omega)} \\
& \quad+\left\|Q_{h}^{H}\left(E_{h} v\right)-E_{h} v\right\|_{L^{2}(\Omega)}+\left\|E_{h} v-v\right\|_{L^{2}(\Omega)} \\
\leq & C H\left|Q_{h}^{H}\left(E_{h} v\right)\right|_{H^{1}(\Omega)}+C H\left|E_{h} v\right|_{H^{1}(\Omega)}+C h|v|_{H^{1}\left(\mathcal{T}_{H}\right)} \\
\leq & C H|v|_{H^{1}\left(\mathcal{T}_{H}\right)} .
\end{aligned}
$$

Therefore, the abstract theory in $\S 2$ is applicable to the case of the scalar P1 nonconforming finite element approximation of the Laplace equation. The generalization to more general symmetric positive definite second-order scalar elliptic problems is straightforward.

Remark. We can also use the P1 conforming finite element space on the coarser grid. Let

$$
\tilde{V}_{H}:=\left\{v \in C(\bar{\Omega}):\left.v\right|_{T} \in \mathcal{P}_{1}(T) \quad \forall T \in \mathcal{T}_{H} \text { and }\left.v\right|_{\partial \Omega}=0\right\} .
$$

Since $\tilde{V}_{H} \subset V_{H}$ we can define $\tilde{I}_{H}^{h}: \tilde{V}_{H} \longrightarrow V_{h}$ to be the natural injection. Assumptions (A.1a) and (A.1b) then become trivial for $\tilde{I}_{H}^{h}$.

Let the operator $\tilde{F}_{H}: W_{H} \longrightarrow \tilde{V}_{H}$ be defined by

$$
\left(\tilde{F}_{H} w\right)(p)=w(p) \quad \text { for all vertices } p \in \mathcal{T}_{H} .
$$

The estimates $(3.9 \mathrm{~b})$ and $(3.10 \mathrm{~b})$ with $F_{H}$ replaced by $\tilde{F}_{H}$ can be established by arguments analogous to those in the proof of Lemma 3.2. Hence, if we define $\tilde{J}_{h}^{H}: V_{h} \longrightarrow \tilde{V}_{H}$ by

$$
\tilde{J}_{h}^{H}:=\tilde{F}_{H} \circ Q_{h}^{H} \circ E_{h},
$$

then assumptions (A.3a) and (A.3b) hold for $\tilde{J}_{h}^{H}$. Therefore, our theory is also applicable for these choices and we recover the results in [8].

\section{The Morley finite element}

In this section we apply the abstract theory to the Morley finite element approximation of the biharmonic equation. Let $V_{h}$ be the Morley finite element space associated with $\mathcal{T}_{h}$. Then $v \in V_{h}$ if and only if it has the following three properties:

(i) $\left.v\right|_{T}$ is quadratic for all $T \in \mathcal{T}_{h}$

(ii) $v$ is continuous at the vertices and vanishes at the vertices along $\partial \Omega$

(iii) $\frac{\partial v}{\partial n}$ is continuous at the midpoints of interelement boundaries and vanishes at the midpoints along $\partial \Omega$.

$V_{H}$ is defined the same way with respect to $\mathcal{T}_{H}$. Members of $V_{h}$ (resp., $V_{H}$ ) are completely determined by their values at vertices of $\mathcal{T}_{h}$ (resp., $\mathcal{T}_{H}$ ) and the values of their normal derivatives at the midpoints of $\mathcal{T}_{h}$ (resp., $\mathcal{T}_{H}$ ). The symmetric positive definite bilinear forms $a_{h}(\cdot, \cdot)$ and $a_{H}(\cdot, \cdot)$ are defined by

$$
a_{h}\left(v_{1}, v_{2}\right):=\sum_{T \in \mathcal{T}_{h}} \int_{T} \sum_{i, j=1}^{2} \frac{\partial^{2} v_{1}}{\partial x_{i} \partial x_{j}} \frac{\partial^{2} v_{2}}{\partial x_{i} \partial x_{j}} d x \quad \forall v_{1}, v_{2} \in V_{h}
$$


and

$$
a_{H}\left(v_{1}, v_{2}\right):=\sum_{T \in \mathcal{T}_{H}} \int_{T} \sum_{i, j=1}^{2} \frac{\partial^{2} v_{1}}{\partial x_{i} \partial x_{j}} \frac{\partial^{2} v_{2}}{\partial x_{i} \partial x_{j}} d x \quad \forall v_{1}, v_{2} \in V_{H} .
$$

The inner product $(\cdot, \cdot)_{h}$ is defined by

$$
\left(v_{1}, v_{2}\right)_{h}:=h^{2} \sum_{p} v_{1}(p) v_{2}(p)+h^{4} \sum_{m} \frac{\partial v_{1}}{\partial n}(m) \frac{\partial v_{2}}{\partial n}(m) \quad \forall v_{1}, v_{2} \in V_{h},
$$

where the summation is over all vertices $p$ and midpoints $m$ of the triangulation $\mathcal{T}_{h}$. The inner product $(\cdot, \cdot)_{H}$ is defined analogously with respect to $\mathcal{T}_{H}$. It follows from a standard calculation using reference elements and a homogeneity argument for almost affine elements (cf. [7]) that

$$
C_{1}\|v\|_{L^{2}(T)}^{2} \leq|T| \sum_{i=1}^{3}\left(v\left(p_{i}\right)\right)^{2}+|T|^{2} \sum_{i=1}^{3}\left(\frac{\partial v}{\partial n}\left(m_{i}\right)\right)^{2} \leq C_{2}\|v\|_{L^{2}(T)}^{2}
$$

for all $v \in \mathcal{P}_{2}(T)$, where $C_{1}, C_{2}$ depend on the shape of $T$. Hence we have

$$
C\|v\|_{L^{2}(\Omega)}^{2} \leq(v, v)_{h} \leq C\|v\|_{L^{2}(\Omega)}^{2} \quad \forall v \in V_{h}
$$

and

$$
C\|v\|_{L^{2}(\Omega)}^{2} \leq(v, v)_{H} \leq C\|v\|_{L^{2}(\Omega)}^{2} \quad \forall v \in V_{H} .
$$

Assumption (A.2) is trivially satisfied. Let $T \in \mathcal{T}_{h}$ and $\Pi$ be the Morley nodal variable interpolation operator from $C^{1}(\bar{T})$ into $\mathcal{P}_{2}(T)$. For $g \in C^{1}(\bar{T})$ and $v \in$ $\mathcal{P}_{2}(T)$ we have by $(4.4)$

$$
\begin{aligned}
\|\Pi(g v)\|_{L^{2}(T)}^{2} & \leq C\left(|T| \sum_{i=1}^{3}\left[g\left(p_{i}\right) v\left(p_{i}\right)\right]^{2}+|T|^{2} \sum_{i=1}^{3}\left(\frac{\partial(g v)}{\partial n}\left(m_{i}\right)\right)^{2}\right) \\
& \leq C\left(\|g\|_{L^{\infty}(T)}^{2}\|v\|_{L^{2}(T)}^{2}+|T|^{2}\|\nabla g\|_{L^{\infty}(T)}^{2} \sum_{i=1}^{3} v\left(m_{i}\right)^{2}\right) .
\end{aligned}
$$

A homogeneity argument shows that

$$
|T| \sum_{i=1}^{3} v\left(m_{i}\right)^{2} \leq C_{3}\|v\|_{L^{2}(T)}^{2} \quad \forall v \in \mathcal{P}_{2}(T),
$$

where $C_{3}$ depends only on the shape of $T$. Assumption (A.4b) now follows from (4.6) and (4.7).

Next, we verify assumption (A.4a). Let $\hat{T}$ be a reference triangle. Since $|\cdot|_{H^{2}(\hat{T})}$ is a norm on the space $\mathcal{P}_{3}(\hat{T}) / \mathcal{P}_{1}(\hat{T})$, and $\zeta \longrightarrow \Pi \zeta-\zeta$ is a well-defined linear map from $\mathcal{P}_{3}(\hat{T}) / \mathcal{P}_{1}(\hat{T})$ into $\mathcal{P}_{3}(\hat{T})$, we have

$$
|\Pi \zeta-\zeta|_{H^{2}(\hat{T})} \leq C_{4}|\zeta|_{H^{2}(\hat{T})} \quad \forall \zeta \in \mathcal{P}_{3}(\hat{T}),
$$


where $C_{4}$ depends only on the shape of the triangle $\hat{T}$. Therefore,

$$
|\Pi \zeta|_{H^{2}(\hat{T})} \leq\left(1+C_{4}\right)|\zeta|_{H^{2}(\hat{T})} \quad \forall \zeta \in \mathcal{P}_{3}(\hat{T}) .
$$

Assumption (A.4a) now follows from (4.8) and a homogeneity argument for almost affine elements.

It remains to define the operators $I_{H}^{h}$ and $J_{h}^{H}$, and to verify assumptions (A.1) and (A.3). As in the case of P1 nonconforming finite elements, we introduce two spaces $W_{h}$ and $W_{H}$, where

$$
W_{h}:=\left\{w \in C^{1}(\bar{\Omega}):\left.w\right|_{T} \in \mathcal{P}_{5}(T) \quad \forall T \in \mathcal{T}_{h} \text { and } w=\frac{\partial w}{\partial n}=0 \text { on } \partial \Omega\right\}
$$

and $W_{H}$ is defined similarly with respect to $\mathcal{T}_{H}$. Note that $W_{h}$ (resp., $W_{H}$ ) contains the Argyris finite element space $\tilde{W}_{h}$ (resp., $\tilde{W}_{H}$ ) whose members are completely determined by the values of their derivatives up to second order at the vertices of $\mathcal{T}_{h}$ (resp., $\mathcal{T}_{H}$ ) and their normal derivatives at the midpoints of $\mathcal{T}_{h}$ (resp., $\mathcal{T}_{H}$ ) (cf. [1]). Note also that $W_{H} \subset W_{h}$ since $\mathcal{T}_{h}$ is a subdivision of $\mathcal{T}_{h}$, but $\tilde{W}_{H} \not \subset \tilde{W}_{h}$.

As in the case of P1 nonconforming finite elements, the operators $I_{H}^{h}$ and $J_{h}^{H}$ are defined through the commutative diagrams in Fig. 1, where $E_{h}: V_{h} \longrightarrow \tilde{W}_{h} \subseteq W_{h}$ (resp., $\left.E_{H}: V_{H} \longrightarrow \tilde{W}_{H} \subseteq W_{H}\right)$ and $F_{h}: W_{h} \longrightarrow V_{h}$ (resp., $F_{H}: W_{H} \longrightarrow V_{H}$ ) are defined as follows:

$$
\begin{cases}\left(E_{h} v\right)(p)=v(p) & \text { for all internal vertices } p \in \mathcal{T}_{h}, \\ \left(\partial^{\alpha} E_{h} v\right)(p)=\text { average of }\left(\partial^{\alpha} v_{i}\right)(p),|\alpha|=1 & \text { for all internal vertices } p \in \mathcal{T}_{h}, \\ \left(\partial^{\alpha} E_{h} v\right)(p)=0,|\alpha|=2 & \text { for all internal vertices } p \in \mathcal{T}_{h}, \\ \left(\frac{\partial}{\partial n} E_{h} v\right)(m)=\frac{\partial v}{\partial n}(m) & \text { for all internal midpoints } m \in \mathcal{T}_{h},\end{cases}
$$

where $v_{i}=\left.v\right|_{T_{i}}$ and $T_{i}$ contains $p$ as a vertex, and

$$
\begin{cases}\left(F_{h} w\right)(p)=w(p) & \text { for all internal vertices } p \in \mathcal{T}_{h} \\ \left(\frac{\partial}{\partial n}\left(F_{h} w\right)\right)(m)=\frac{\partial w}{\partial n}(m) & \text { for all internal midpoints } m \in \mathcal{T}_{h}\end{cases}
$$

The nodal values of $E_{h} v$ are zero along $\partial \Omega$. The definitions of $E_{H}$ and $F_{h}$ are the same with respect to $\mathcal{T}_{H}$.

The operators $I_{H}^{h}: V_{H} \longrightarrow V_{h}$ and $J_{h}^{H}: V_{h} \longrightarrow V_{H}$ are then defined by

$$
I_{H}^{h}:=F_{h} \circ E_{H}
$$

and

$$
J_{h}^{H}:=F_{H} \circ Q_{h}^{H} \circ E_{h},
$$

where $Q_{h}^{H}: W_{h} \longrightarrow W_{H}$ is the $L^{2}$-orthogonal projection operator.

Again, note that $I_{H}^{h}$ is represented by a sparse, banded matrix with respect to the natural nodal bases of $V_{h}$ and $V_{H}$. 
Lemma 4.1. The following estimates on $Q_{h}^{H}$ hold:

$$
\begin{aligned}
& \left|Q_{h}^{H} w\right|_{H^{2}(\Omega)} \leq C|w|_{H^{2}(\Omega)}, \\
& \quad\left\|w-Q_{h}^{H} w\right\|_{L^{2}(\Omega)}+H\left|w-Q_{h}^{H} w\right|_{H^{1}(\Omega)} \leq C H^{2}|w|_{H^{2}(\Omega)},
\end{aligned}
$$

for all $w \in W_{h}$.

Proof. Let $w \in W_{h}$. By Theorem 4.1.2 in [19], there exits $w^{\prime} \in W_{H}$ such that

$$
\left|w-w^{\prime}\right|_{H^{s}(\Omega)} \leq C H^{2-s}|w|_{H^{2}(\Omega)}, \quad s=0,1,2 .
$$

Let $Q_{H}: L^{2}(\Omega) \longrightarrow W_{H}$ be the $L^{2}$-orthogonal projection operator (hence $Q_{h}^{H}=$ $\left.\left.Q_{H}\right|_{W_{h}}\right)$. By (4.15) and standard inverse estimates, we have for $s=0,1,2$,

$$
\begin{aligned}
\left|w-Q_{h}^{H}\right|_{H^{s}(\Omega)} & \leq\left|w-w^{\prime}\right|+\left|Q_{H}\left(w^{\prime}-w\right)\right|_{H^{s}(\Omega)} \\
& \leq C H^{2-s}|w|_{H^{2}(\Omega)}+C H^{-s}\left\|Q_{H}\left(w^{\prime}-w\right)\right\|_{L^{2}(\Omega)} \\
& \leq C H^{2-s}|w|_{H^{2}(\Omega)}+C H^{-s}\left\|\left(w^{\prime}-w\right)\right\|_{L^{2}(\Omega)} \\
& \leq C H^{2-s}|w|_{H^{2}(\Omega)} .
\end{aligned}
$$

The estimates (4.13) and (4.14) follow immediately from (4.16).

The proof of the following lemma is similar to the proof of Lemma 3.2 and is therefore omitted.

Lemma 4.2. The following estimates on $F_{h}$ and $F_{H}$ hold:

$$
\begin{aligned}
& \left|F_{h} w\right|_{H^{2}\left(\mathcal{T}_{h}\right)} \leq C|w|_{H^{2}(\Omega)}, \\
& \left|F_{H} \tilde{w}\right|_{H^{2}\left(\mathcal{T}_{H}\right)} \leq C|\tilde{w}|_{H^{2}(\Omega)}, \\
& \left\|w-F_{h} w\right\|_{L^{2}(\Omega)}+h\left|w-F_{h} w\right|_{H^{1}\left(\mathcal{T}_{h}\right)} \leq C h^{2}|w|_{H^{2}(\Omega)}, \\
& \left\|\tilde{w}-F_{H} \tilde{w}\right\|_{L^{2}(\Omega)}+H\left|\tilde{w}-F_{H} \tilde{w}\right|_{H^{1}\left(\mathcal{T}_{H}\right)} \leq C H^{2}|\tilde{w}|_{H^{2}(\Omega)},
\end{aligned}
$$

for all $w \in W_{h}$ and $\tilde{w} \in W_{H}$.

Lemma 4.3. The following estimates on $E_{h}$ and $E_{H}$ hold:

$$
\begin{aligned}
& \left|E_{h} v\right|_{H^{2}(\Omega)} \leq C|v|_{H^{2}\left(\mathcal{T}_{h}\right)}, \\
& \left|E_{H} \tilde{v}\right|_{H^{2}(\Omega)} \leq C|\tilde{v}|_{H^{2}\left(\mathcal{T}_{H}\right)}, \\
& \left\|v-E_{h} v\right\|_{L^{2}(\Omega)}+h\left|v-E_{h} v\right|_{H^{1}\left(\mathcal{T}_{h}\right)} \leq C h^{2}|v|_{H^{2}\left(\mathcal{T}_{h}\right)}, \\
& \left\|\tilde{v}-E_{H} \tilde{v}\right\|_{L^{2}(\Omega)}+H\left|\tilde{v}-E_{H} \tilde{v}\right|_{H^{1}\left(\mathcal{T}_{H}\right)} \leq C H^{2}|\tilde{v}|_{H^{2}\left(\mathcal{T}_{H}\right)},
\end{aligned}
$$

for all $v \in V_{h}$ and $\tilde{v} \in V_{H}$.

Proof. It suffices to establish (4.19a) and (4.20a). Let $v \in V_{h}, T \in \mathcal{T}_{h}, w=\left.v\right|_{T}$ and $\tilde{w}=\left.\left(E_{h} v\right)\right|_{T}$. The two functions $w, \tilde{w} \in \mathcal{P}_{5}(T)$ are related by

$$
w-\tilde{w}=\sum_{i=1}^{3} \sum_{|\alpha|=1,2} \partial_{\alpha}(w-\tilde{w})\left(p_{i}\right) r_{\alpha, i}
$$




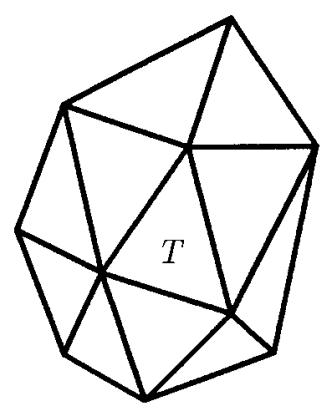

FiguRE 4

where the points $p_{i}$ are the vertices of $T$, and the functions $r_{\alpha, i}$ are the nodal basis functions corresponding to the nodal variables $\left(\partial_{\alpha} v\right)\left(p_{i}\right)$ of the Argyris finite element. The following estimates are obtained by the standard techniques of almost affine family of finite elements (cf. [7]):

$$
\begin{array}{ll}
\left\|r_{\alpha, i}\right\|_{L^{2}(T)} \leq C(T) h_{T}^{2} & \text { for }|\alpha|=1, \\
\left\|r_{\alpha, i}\right\|_{L^{2}(T)} \leq C(T) h_{T}^{3} & \text { for }|\alpha|=2,
\end{array}
$$

where $h_{T}=\operatorname{diam} T$, and $C(T)$ represents a generic positive constant which depends continuously on the minimum angle of the triangle $T$. By a standard inverse estimate and (4.9) we have

$$
\begin{aligned}
\left|\partial_{\alpha}(w-\tilde{w})\left(p_{i}\right)\right| & =\left|\partial_{\alpha} w\left(p_{i}\right)\right| \\
& \leq|v|_{W_{\infty}^{2}(T)} \\
& \leq C(T) h_{T}^{-1}|v|_{H^{2}(T)}
\end{aligned}
$$

for $|\alpha|=2$.

Recall from (4.9) that for $|\alpha|=1,\left[\partial_{\alpha}\left(E_{h} v\right)\right](p)=$ average of $\partial_{\alpha} v_{j}(p)$, where $v_{j}=\left.v\right|_{T_{j}}$ and $T_{j}$ contains $p$ as a vertex. Suppose $T_{1}$ and $T_{2}$ are two triangles in $\mathcal{T}_{h}$ sharing the common edge $e$ which contains $p$ as an endpoint. Since $\left.v\right|_{T_{1}}$ and $\left.v\right|_{T_{2}}$ agree at the two endpoints of $e$, the difference of $\partial_{s}\left(\left.v\right|_{T_{1}}\right)$ and $\partial_{s}\left(\left.v\right|_{T_{2}}\right)(s$ is the arc length along $e$ ) at $p$ is bounded by $(|e| / 2)\left[|v|_{W_{\infty}^{2}\left(T_{1}\right)}+|v|_{W_{\infty}^{2}\left(T_{2}\right)}\right]$.

Similarly, since the normal derivatives of $\left.v\right|_{T_{1}}$ and $\left.v\right|_{T_{2}}$ at the midpoint $m$ of $e$ agree, the difference of $\partial_{n}\left(\left.v\right|_{T_{1}}\right)$ and $\partial_{n}\left(\left.v\right|_{T_{2}}\right)$ ( $n$ is a normal of $e$ ) at $p$ is bounded by $(|e| / 2)\left[|v|_{W_{\infty}^{2}\left(T_{1}\right)}+|v|_{W_{\infty}^{2}\left(T_{2}\right)}\right]$.

Therefore, we have the following estimate:

$$
\begin{aligned}
\sum_{i=1}^{3} \sum_{|\alpha|=1}\left|\partial_{\alpha}(w-\tilde{w})\left(p_{i}\right)\right| & \leq k_{T} \sum_{T^{\prime}} h_{T^{\prime}}\left|\left(\left.v\right|_{T^{\prime}}\right)\right|_{W_{\infty}^{2}\left(T^{\prime}\right)} \\
& \leq k_{T} \sum_{T^{\prime}} C\left(T^{\prime}\right)\left|\left(\left.v\right|_{T^{\prime}}\right)\right|_{H^{2}\left(T^{\prime}\right)},
\end{aligned}
$$

where the summation is over all the triangles $T^{\prime}$ which share at least one vertex with $T$ (cf. Fig. 4), and $k_{T}$ is a constant which depends only on the total number of such $T^{\prime}$. 
Combining (4.21)-(4.25) and using the quasi-uniformity of $\mathcal{T}_{h}$, we have

$$
\left\|v-E_{h} v\right\|_{L^{2}(T)} \leq C h^{2} \sum_{T^{\prime}}|v|_{H^{2}\left(T^{\prime}\right)} .
$$

Note that estimate (4.26) also holds if some of the vertices of $T$ belong to $\partial \Omega$. Summing up the square of (4.26) over all the triangles $T$ in $\mathcal{T}_{h}$, we obtain

$$
\left\|v-E_{h} v\right\|_{L^{2}(\Omega)} \leq C h^{2}|v|_{H^{2}\left(\mathcal{T}_{h}\right)} .
$$

The rest of the estimates in (4.19a) and (4.20a) now follow from standard inverse estimates and the triangle inequality.

The following proposition follows from Lemmas 4.1-4.3, just as Proposition 3.1 followed from Lemmas 3.1-3.3.

Proposition 4.1. Assumptions (A.1) and (A.3) hold for $I_{H}^{h}$ and $J_{h}^{H}$ defined by (4.11) and (4.12), respectively.

\section{Divergence-free P1 nonconforming Finite Element}

In this section we adapt the abstract theory to the divergence-free P1 nonconforming finite element approximation of the stationary Stokes equations. We assume that $\Omega$ is simply connected (i.e., flow without obstacle). The case where there are obstacles is more complicated and is discussed elsewhere (cf. [5]). Throughout this section we use undertildes to denote vector-valued functions and operators. The operators curl and div are given by

$$
\begin{aligned}
& \underset{\sim}{\operatorname{curl} p}=\left(\begin{array}{r}
\partial p / \partial x_{2} \\
-\partial p / \partial x_{1}
\end{array}\right), \\
& \operatorname{div} \underset{\sim}{v}=\frac{\partial v_{1}}{\partial x_{1}}+\frac{\partial v_{2}}{\partial x_{2}} .
\end{aligned}
$$

The finite element space $V_{h}$ is defined by

$$
\begin{aligned}
V_{h}:= & \left\{\underset{\sim}{v} \in \underset{\sim}{L^{2}}(\Omega): \underset{\sim}{v} \in \underset{\sim}{\mathcal{P}_{1}}(T) \quad \forall T \in \mathcal{T}_{h}, \underset{\sim}{v}\right. \text { is continuous } \\
& \text { at the midpoints of interelement boundaries, } \underset{\sim}{v} \text { vanishes at the } \\
& \text { midpoints along } \left.\partial \Omega, \text { and } \operatorname{div}\left(\left.\underset{\sim}{v}\right|_{T}\right)=0 \quad \forall T \in \mathcal{T}_{h}\right\} .
\end{aligned}
$$

$V_{H}$ is defined the same way with respect to $\mathcal{T}_{H}$.

The symmetric positive definite bilinear forms $a_{h}(\cdot, \cdot)$ and $a_{H}(\cdot, \cdot)$ are given by

$$
a_{h}(\underset{\sim}{v}, \underset{\sim}{w}):=\sum_{T \in \mathcal{T}_{h}} \int_{T} \sum_{i, j=1}^{2} \frac{\partial v_{i}}{\partial x_{j}} \frac{\partial w_{i}}{\partial x_{j}} d x \quad \forall \underset{\sim}{v}, \underset{\sim}{w} \in V_{h}
$$

and

$$
a_{H}(\underset{\sim}{v}, \underset{\sim}{w}):=\sum_{T \in \mathcal{T}_{H}} \int_{T} \sum_{i, j=1}^{2} \frac{\partial v_{i}}{\partial x_{j}} \frac{\partial w_{i}}{\partial x_{j}} d x \quad \forall \underset{\sim}{v} \underset{\sim}{w} \in V_{H} .
$$



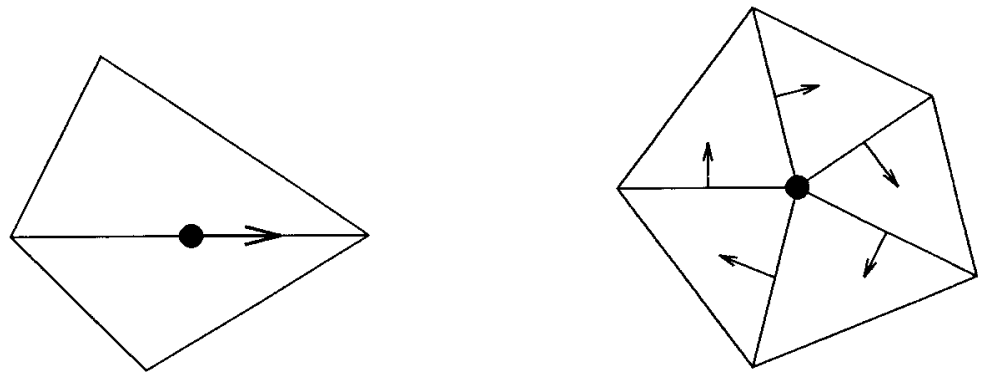

FiguRE 5

In order to define the inner products $(\cdot, \cdot)_{h}$ and $(\cdot, \cdot)_{H}$, we must first describe the bases of $V_{h}$ and $V_{H}$.

Let $e$ be an edge in $\mathcal{T}_{h}$. Denote by $\phi_{e}$ the piecewise linear function on $\Omega$ (with respect to $\mathcal{T}_{h}$ ) that takes the value 1 at the midpoint of the edge $e$ and 0 at all other midpoints.

The first kind of basis function is associated with internal edges. Let

$$
\underset{\sim}{\mu_{e}}:=\phi_{e} t_{\sim},
$$

where $e$ is an internal edge and $t_{e}$ is a unit vector tangential to $e$ (cf. Fig. 5).

The second kind of basis function is associated with internal vertices. Let $p$ be an internal vertex and let $e_{1}, e_{2}, \cdots, e_{\ell}$ be the edges in $\mathcal{T}_{h}$ that have $p$ as an endpoint. Let

$$
\nu_{p}:=\sum_{i=1}^{\ell}\left|e_{i}\right|^{-1} \phi_{e_{i}} \underset{\sim}{n_{e_{i}}}
$$

where $n_{e_{i}}$ is a unit vector normal to $e_{i}$ pointing in the clockwise direction (cf. Fig. 5). Then $B_{h}:=\left\{\mu_{e}: e\right.$ is an internal edge of $\left.\mathcal{T}_{h}\right\} \cup\left\{\nu_{p}: p\right.$ is an internal vertex of $\left.\mathcal{T}_{h}\right\}$ is a basis of $V_{h}$ (cf. [16]). The basis $B_{H}$ (resp., $B_{j}$ ) of $V_{H}$ (resp., $V_{j}$ ) is defined similarly.

Let $\underset{\sim}{v}=\sum a_{i} \mu_{e_{i}}+\sum b_{j} \nu_{p_{j}}$ and $\underset{\sim}{w}=\sum \alpha_{i} \mu_{e_{i}}+\sum \beta_{j} \nu_{p_{j}}$ be two members of $V_{h}$, where the summations are taken over all internal edges and all internal vertices. Then $(\underset{\sim}{v}, \underset{\sim}{w})_{h}$ is defined by

$$
(\underset{\sim}{v} \underset{\sim}{w})_{h}:=h^{2} \sum a_{i} \alpha_{i}+h^{4} \sum b_{j} \beta_{j}
$$

The inner product $(\cdot, \cdot)_{H}$ is defined similarly.

The theory developed in $\S 2$ cannot be directly applied to the problem here because of the divergence-free constraint. To be more specific, the $v_{j}$ 's defined in the proof of Lemma 2.3 do not satisfy the divergence-free constraint.

We will modify the theory in the following manner. We establish assumptions (A.1a) and (A.2), and then Lemmas 2.1 and 2.2 remain valid. We will then prove Lemma 2.3 directly by exploiting the connection between the divergence-free P1 
nonconforming finite element and the Morley finite element. Since Lemma 2.4 remains unchanged, Theorem 2.1 then holds.

Assumption (A.2) is trivially true. In order to define $I_{H}^{h}$ and verify assumptions (A.1a) and (A.1b), we need to consider the connection between $V_{h}$ (resp., $V_{j}, V_{H}$ ) and the Morley finite element spaces defined in $\S 4$, which we denote by $M_{h}$ (resp., $\left.M_{j}, M_{H}\right)$ here.

There is an isomorphism between $M_{h}$ and $V_{h}$ (cf. [12]) given by the operator $\operatorname{curl}_{h}$, where

$$
\left.\left(\operatorname{curl}_{h} \psi\right)\right|_{T}=\operatorname{curl}_{\sim}\left(\left.\psi\right|_{T}\right) \quad \forall T \in \mathcal{T}_{h} .
$$

The isomorphism $\operatorname{curl}_{H}: M_{H} \longrightarrow V_{H}$ is defined similarly. Note that $\left.\operatorname{curl}_{h}\right|_{M_{j}}$ is an isomorphism from $\widetilde{M}_{j}$ onto $V_{j}$. The inverse of $\underset{\sim}{\operatorname{curl}_{h}}\left(\operatorname{resp} ., \operatorname{curl}_{H}\right)$ will be denoted by $\operatorname{curl}_{h}^{-1}$ (resp., $\operatorname{curl}_{H}^{-1}$ ).

In terms of the basis $B_{h}$ of $V_{h}$, we have a simple description of $\operatorname{curl}_{h}$. Let $\psi \in M_{h}$. Then we have

$$
\operatorname{curl}_{h} \psi=\sum a_{i} \mu_{\sim} e_{i}+\sum b_{j} \nu_{p_{j}}
$$

where $a_{i}=\frac{\partial \psi}{\partial n_{e_{i}}}\left(m_{i}\right), m_{i}$ is the midpoint of edge $e_{i}, \underset{\sim}{t_{e_{i}}}$ is obtained by rotating $\underset{\sim}{n_{e_{i}}}$ clockwise through a right angle, and $b_{j}=\psi\left(p_{j}\right)$.

We are now ready to define the operator $I_{H}^{h}$. Let $\tilde{I}_{H}^{h}$ be the intergrid transfer operator between the Morley spaces $M_{H}$ and $M_{h}$ defined in $\S 4$. The operator $I_{H}^{h}: V_{H} \longrightarrow V_{h}$ is defined by

$$
I_{H}^{h}:=\operatorname{curl}_{h} \circ \tilde{I}_{H}^{h} \circ \operatorname{curl}_{H}^{-1} .
$$

The relations between these operators are illustrated by the commutative diagram in Fig. 6.

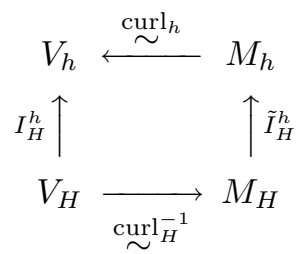

Figure 6

In view of $(5.7), I_{H}^{h}$ is represented by a sparse banded matrix with respect to the bases $B_{h}$ and $B_{H}$.

Note that we have the trivial identities

$$
\left|\operatorname{curl}_{h} \psi\right|_{H^{1}\left(\mathcal{T}_{h}\right)}=|\psi|_{H^{2}\left(\mathcal{T}_{h}\right)}, \quad\left\|\operatorname{curl}_{h} \psi\right\|_{L^{2}(\Omega)}=|\psi|_{H^{1}\left(\mathcal{T}_{h}\right)}
$$

and

$$
\left|\operatorname{curl}_{\sim} \phi\right|_{H^{1}\left(\mathcal{T}_{H}\right)}=|\phi|_{H^{2}\left(\mathcal{T}_{H}\right)}, \quad\left\|\operatorname{curl}_{\sim} \phi\right\|_{L^{2}(\Omega)}=|\phi|_{H^{1}\left(\mathcal{T}_{H}\right)},
$$


where $\psi$ (resp., $\phi$ ) is piecewise $C^{1}$ with respect to $\mathcal{T}_{h}$ (resp., $\mathcal{T}_{H}$ ).

Lemma 5.1. Assumption (A.1a) (for $k=1$ ) holds for $I_{H}^{h}$ defined by (5.8).

Proof. It was established in Proposition 4.1 that $\tilde{I}_{H}^{h}$ has the following property

$$
\left|\tilde{I}_{H}^{h} \psi\right|_{H^{2}\left(\mathcal{T}_{h}\right)} \leq C|\psi|_{H^{2}\left(\mathcal{T}_{H}\right)} \quad \forall \psi \in M_{H}
$$

Assumption (A.1a) follows immediately from (5.8), (5.9) and (5.10).

Finally, we establish Lemma 2.3 in the present context.

Lemma 5.2. Given any $\underset{\sim}{v} \in V_{h}$, there exists $\underset{\sim}{v_{0}} \in V_{H},{\underset{\sim}{v}}_{j} \in V_{j}(1 \leq j \leq J)$ such that

$$
\underset{\sim}{v}=I_{H}^{h}{\underset{\sim}{0}}_{0}+\sum_{j=1}^{J}{\underset{\sim}{j}}_{j}
$$

and

$$
a_{H}\left({\underset{\sim}{0}}_{0},{\underset{\sim}{0}}_{0}\right)+\sum_{j=1}^{J} a_{h}\left(v_{\sim},{\underset{\sim}{j}}_{j}\right) \leq C N_{c}\left(1+\left(\frac{H}{\delta}\right)^{4}\right) a_{h}(\underset{\sim}{v}, \underset{\sim}{v}) .
$$

Proof. Let $\psi=\operatorname{curl}_{h}^{-1} \underset{\sim}{v} \in M_{h}$. It follows from Proposition 4.1 that Lemma 2.3 holds for the Morley finite element spaces. Therefore, there exists $\psi_{0} \in M_{H}, \psi_{j} \in M_{j}$ $(1 \leq j \leq J)$ such that

$$
\psi=\tilde{I}_{H}^{h} \psi_{0}+\sum_{j=1}^{J} \psi_{j}
$$

and

$$
\left|\psi_{0}\right|_{H^{2}\left(\mathcal{T}_{H}\right)}^{2}+\sum_{j=1}^{J}\left|\psi_{j}\right|_{H^{2}\left(\mathcal{T}_{h}\right)}^{2} \leq C N_{c}\left(1+\left(\frac{H}{\delta}\right)^{4}\right)|\psi|_{H^{2}\left(\mathcal{T}_{h}\right)}^{2} .
$$

Let ${\underset{\sim}{v}}_{0}=\operatorname{curl}_{\sim} \psi_{0}$ and ${\underset{\sim}{v}}_{j}=\operatorname{curl}_{\sim} \psi_{j}$ for $1 \leq j \leq J$. Then, using (5.8) and (5.13), we obtain

$$
\begin{aligned}
& \underset{\sim}{v}=\operatorname{curl}_{h} \psi \\
& =\operatorname{curl}_{\sim}\left(\tilde{I}_{H}^{h} \psi_{0}+\sum_{j=1}^{J} \psi_{j}\right)
\end{aligned}
$$

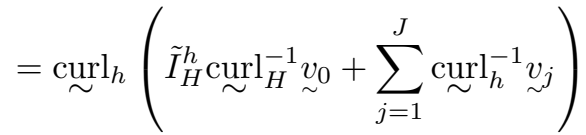

$$
\begin{aligned}
& =I_{H}^{h}{\underset{\sim}{v}}_{0}+\sum_{j=1}^{J} v_{\sim} .
\end{aligned}
$$

The estimate (5.14) can be rewritten, using (5.9), as

$$
\left|\underset{\sim}{v_{0}}\right|_{H^{1}\left(\mathcal{T}_{H}\right)}^{2}+\sum_{j=1}^{J}\left|v_{\sim}\right|_{H^{1}\left(\mathcal{T}_{h}\right)}^{2} \leq C N_{c}\left(1+\left(\frac{H}{\delta}\right)^{4}\right)|\underset{\sim}{v}|_{H^{1}\left(\mathcal{T}_{h}\right)}^{2},
$$

which is equivalent to (5.14) by (A.2).

As was pointed out earlier, Lemmas 5.1 and 5.2 yield the following theorem. 
Theorem 5.1. The two-level additive Schwarz preconditioner $B$ for the divergencefree P1 nonconforming finite element method defined by (2.16) satisfies

$$
\frac{\lambda_{\max }\left(B A_{h}\right)}{\lambda_{\min }\left(B A_{h}\right)} \leq C \frac{\omega_{1}}{\omega_{0}} N_{c}^{2}\left(1+\left(\frac{H}{\delta}\right)^{4}\right)
$$

where $A_{h}$ is the operator respresenting the discretized stationary Stokes equations.

\section{REFERENCES}

1. J.H. Argyris, I. Fried, and D.W. Scharpf, The TUBA family of plate elements for the matrix displacement method, Aero. J. Roy. Aero. Soc. 72 (1968), 701-709.

2. J.H. Bramble and J. Xu, Some estimates for a weighted $L^{2}$ projection, Math. Comp. 56 (1991), 463-476. MR 91k:65140

3. S.C. Brenner, Two-level additive Schwarz preconditioners for nonconforming finite elements, Domain Decomposition Methods in Scientific and Engineering Computing, Contemporary Mathematics 180 (D.E. Keyes et al., eds.), American Mathematical Society, Providence, 1994, pp. 9-14. MR 95j:65134

4. _ A two-level additive Schwarz preconditioner for nonconforming plate elements, Numer. Math. 72 (1996), 419-447.

5. - A two-level additive Schwarz preconditioner for the stationary Stokes equations, Adv. Comp. Math. 4 (1995), 111-126.

6. S.C. Brenner and L.R. Scott, The Mathematical Theory of Finite Element Methods, SpringerVerlag, New York, 1994. MR 95f:65001

7. P.G. Ciarlet, The Finite Element Method for Elliptic Problems, North-Holland, AmsterdamNew York-Oxford, 1978. MR 58:25001

8. L.C. Cowsar, Domain decomposition methods for nonconforming finite elements spaces of Lagrange-type, Proceedings of the Sixth Copper Mountain Conference on Multigrid Methods, NASA Conference Publication 3224 (1993), 93-109.

9. M. Crouzeix and P.-A. Raviart, Conforming and nonconforming finite element methods for solving the stationary Stokes equations I, R.A.I.R.O. R-3 (1973), 33-75. MR 49:8401

10. M. Dryja and O.B. Widlund, An additive variant of the Schwarz alternating method in the case of many subregions, Technical Report 339, Department of Computer Science, Courant Institute (1987).

11. _ Some domain decomposition algorithms for elliptic problems, Technical Report 438, Department of Computer Science, Courant Institute (1989).

12. R.S. Falk and M.E. Morley, Equivalence of finite element methods for problems in elasticity, SIAM J. Numer. Anal. 27 (1990), 1486-1505. MR 91i:65177

13. L.S.D. Morley, The triangular equilibrium problem in the solution of plate bending problems, Aero. Quart. 19 (1968), 149-169.

14. S.V. Nepomnyaschikh, On the application of the bordering method to the mixed boundary value problem for elliptic equations and on mesh norms in $W_{2}^{1 / 2}(S)$, Sov. J. Numer. Anal. Math. Modelling 4 (1989), 493-506.

15. M. Sarkis, Two-level Schwarz methods for nonconforming finite elements and discontinuous coefficients, Proceedings of the Sixth Copper Mountain Conference on Multigrid Methods, NASA Conference Publication 3224 (1993), 543-565.

16. F. Thomasset, Implementation of Finite Element Methods for Navier-Stokes Equations, Springer-Verlag, New York, 1981. MR 84k:76015

17. O.B. Widlund, Some Schwarz methods for symmetric and nonsymmetric elliptic problems, Fifth International Symposium on Domain Decomposition Methods for Partial Differential Equations (D.E. Keyes et al., eds.), SIAM, Philadelphia, 1991, pp. 19-36.

18. J. Xu, Iterative methods by space decomposition and subspace correction, SIAM Review 34 (1992), 581-613. MR 93k:65029 
19. X. Zhang, Studies in Domain Decomposition: Multi-level Methods and the Biharmonic Dirichlet Problem, Dissertation, (Technical Report 584, Department of Computer Science) Courant Institute (1991).

Department of Mathematics and Computer Science, Clarkson University, Potsdam, New YoRK 13699-5815

Current address: Department of Mathematics, University of South Carolina, Columbia, South Carolina 29208

E-mail address: brenner@math.sc.edu 